\title{
Berberine ameliorates neonatal necrotizing enterocolitis by activating the phosphoinositide 3-kinase/protein kinase B signaling pathway
}

\author{
CHENGZHI FANG, LILI XIE, CHUNMEI LIU, CHUNHUA FU, WEI YE, HONG LIU and BINGHONG ZHANG \\ Department of Neonatology, Renmin Hospital of Wuhan University, Wuhan, Hubei 430060, P.R. China
}

Received October 16, 2016; Accepted August 22, 2017

DOI: $10.3892 /$ etm.2018.5858

\begin{abstract}
Neonatal necrotizing enterocolitis (NEC) is a severe acquired disease that predominantly affects the small intestine of neonates. NEC is caused by a combination of metabolic products, dysfunctions of the blood vessels, mucus and other unknown factors. Berberine may induce beneficial effects on necrotic and cardiovascular diseases due to its anti-inflammatory and anti-apoptotic effects on epithelial cells. In the present study, the therapeutic effects of berberine were investigated and the potential mechanisms by which it functions within a neonatal NEC mouse model were analyzed. Inflammation and levels of associated factors were measured in the serum of mice with NEC prior to and following treatment with berberine. Apoptotic rates in epithelial cells were analyzed following treatment with berberine. The expression of genes associated with apoptosis and apoptosis signaling were determined in epithelial cells in the small intestines of mice with NEC following treatment with berberine. The phosphoinositide 3-kinase (PI3K)/protein kinase B (AKT) signaling pathway was investigated in epithelial cells isolated from mice following treatment with either berberine or PBS. Histology and immunohistochemistry were used to determine the area of infarction and apoptosis. Body weight and food intake were measured to evaluate the physical effects of berberine on mice with NEC. The results indicated that berberine attenuated the inflammation caused by NEC in mice after 10 days of treatment. The apoptosis rate of epithelial cells isolated from experimental mice was decreased following berberine treatment. Western blot analysis indicated that the expression of the anti-apoptotic genes c-Myc and p53 were upregulated by berberine, whereas caspase-3 and -9 levels were downregulated in epithelial cells following treatment
\end{abstract}

Correspondence to: Professor Binghong Zhang, Department of Neonatology, Renmin Hospital of Wuhan University, 99 Zhizhidong Road, Wuhan, Hubei 430060, P.R. China

E-mail: zhangbinghongdoc@163.com

Key words: neonatal necrotizing enterocolitis, berberine, inflammation, apoptosis, phosphoinositide 3 kinase/protein kinase B signaling pathway with berberine. In addition, the expression and phosphorylation levels of PI3K and AKT were downregulated in epithelial cells following treatment with berberine. An in vitro assay indicated that treatment with PI3K alone increased the expression of AKT and promoted the apoptosis of epithelial cells. Treatment with berberine markedly increased epidermal growth factor (EGF) and Bcl-2 expression levels, the activity of epithelial cells and decreased the infarction area of the small intestine. Accordingly, the body weight and food intake of mice with NEC were increased following berberine treatment. Therefore, the results of the present study demonstrate that berberine inhibits inflammation and apoptosis via the PI3K/AKT signaling pathway and may therefore attenuate the progression of NEC. These results suggest that berberine may be a potential therapeutic agent for the treatment of patients with NEC.

\section{Introduction}

Neonatal necrotizing enterocolitis (NEC) is a severe acquired disease that causes mucosal damage and necrosis in the bowel. NEC most frequently occurs in premature and sick infants, with an incidence of $1.5-7.2 \%$, and its development is influenced by many different factors (1). It has been demonstrated that ischemic hypoxia leads to localized necrosis or necrosis of the entire intestine and colon (2). Abdominal distension and hematochezia are the primary symptoms of neonatal NEC, which is characterized by necrosis of the intestinal mucosa and may also lead to systemic sepsis and multi-organ failure (3). Necrosis of the intestinal tract significantly compromises the function of the digestive system and results in a loss of appetite and weight loss (4). Additionally, previous studies have indicated that inflammation and apoptosis are associated with disease progression in patients with neonatal NEC $(5,6)$. Therefore, attenuating inflammation and inhibiting the apoptosis of epithelial cells in the small intestine are potential methods of treating neonatal NEC.

Inflammation is one of the most common characteristics of NEC and serves a key role in the progression of necrotic diseases (7). It has been demonstrated that inflammation is a potential pathophysiological mechanism that contributes to the development of neonatal NEC, as it induces the excessive production of pro-inflammatory cytokines (8). Chan et al (9) proposed that blocking LPS activation and 
leukocyte infiltration may be a potential method of treating lipopolysaccharide (LPS)/cluster of differentiation 14/toll like receptor 4 (TLR4)-mediated inflammation. A previous review systematically elaborated on the pathogenesis and therapeutic implications of bacterial infection, intestinal barrier function and inflammatory responses of immature enterocytes (2). Furthermore, a number of studies have demonstrated that inhibiting intestinal inflammation contributes to the improvement of neonatal NEC and have analyzed the potential of factors involved in inflammatory signaling pathways as therapeutic targets $(10,11)$. It has previously been demonstrated that berberine attenuates LPS-induced inflammation and extracellular matrix accumulation by regulating the nuclear factor (NF)- $\mathrm{B}$ signaling pathway (12). Therefore, the present study hypothesized that berberine may be beneficial in the treatment of neonatal NEC and the anti-inflammatory effect of berberine in a mouse model was investigated.

Apoptosis of epithelial cells in the small intestine is the most common characteristic observed in patients with NEC (13). It has been reported that intestinal epithelial apoptosis initiates gross bowel necrosis and leads to anabrosis in an experimental rat model of NEC (14). In an experimental model of NEC, epidermal growth factor (EGF) and the apoptosis regulator $\mathrm{B}$-cell lymphoma $(\mathrm{Bcl})-2$ reduced the apoptosis of intestinal epithelial cells and protected the intestinal epithelium against NEC injury (15). In addition, it has been demonstrated that decreasing intestinal apoptosis improves neonatal NEC in rats via the heparin-binding EGF-like growth factor (16). Furthermore, berberine attenuated ischemia/reperfusion-induced myocardial apoptosis by activating 5 ' adenosine monophosphate-activated protein kinase and the phosphoinositide 3-kinase (PI3K)/protein kinase B (AKT) signaling pathways in a diabetic rat model (17).

In the present study, the anti-inflammatory and anti-apoptotic effects of berberine in the intestinal epithelium of a neonatal NEC mouse model were investigated. The PI3K/AKT signaling pathway in epithelial cells was investigated as the potential mechanism by which berberine alleviates neonatal NEC. The results revealed that treatment with berberine increased EGF expression, the activity of epithelial cells and decreased the area of infarction within the small intestine. These results suggest that berberine may be a potential therapeutic agent for the treatment of patients with NEC.

\section{Materials and methods}

Ethics statement. The present study was conducted in accordance with the Guide for the Care and Use of Laboratory Animals by Renmin Hospital of Wuhan University (Hubei, China) (18). All surgeries and experiments were performed under anesthetic to minimize any pain caused to the animal. The present study was approved by the Ethics Committee of Renmin Hospital of Wuhan University.

Animals. A neonatal NEC mouse model was established following a previously described protocol (19). A total of 40 postnatal 4-day-old male C57BL/6JCnc mice (weight, 5-10 g; Beijing Vital River Laboratory Animal Technology, Beijing, China) were used. All mice were housed in a temperature and humidity controlled room at $25 \pm 1^{\circ} \mathrm{C}$ and $55 \pm 5 \%$, respectively, with an artificial $12 \mathrm{~h}$ light/dark cycle and they had free access to food and water. To establish a neonatal NEC model, mice were administered $150 \mathrm{mmol} / \mathrm{l}$ acetic acid by enema following induction of anesthesia with sodium pentobarbital $(40 \mathrm{mg} / \mathrm{kg}$; China National Pharmaceutical Group Corporation, Beijing, China) by intraperitoneal injection. Mice were then separated into two groups (15 mice/group) and received oral treatment of berberine $(5 \mathrm{mg} / \mathrm{ml})$ or PBS $(5 \mathrm{mg} / \mathrm{ml})$ once a day for 10 days. The body weights and food intake of experimental mice were measured prior to each injection. All mice were sacrificed on day 10 for histological analysis.

Cell culture. Epithelial cells from the small intestine were isolated from the 10 experimental mice, and cultured in Dulbecco's modified Eagle's medium with $10 \%$ fetal bovine serum (both from Gibco; Thermo Fisher Scientific, Inc., Waltham, MA, USA). Epithelial cells were cultured in a $5 \% \mathrm{CO}_{2}$ humidified atmosphere at $37^{\circ} \mathrm{C}$, following the attachment of the cells, passage 1 epithelial cells were treated with PI3K (5 mg/ml) (Promega Corporation, Madison, WI, USA) and/or berberine (Xi'an Le Sen Bio-Technology Co., Ltd., Xi'an, China) $(5 \mathrm{mg} / \mathrm{ml})$ for $24 \mathrm{~h}$ to analyze the effects of PI3K on apoptosis and the expression of apoptosis-related proteins.

ELISA assay. ELISA kits were used to determine serum levels of myeloid differentiation protein-2 (MD-2; cat. no. MBS261609; MyBioSource, Inc., San Diego, CA, USA), NF- $\kappa$ B (cat. no. ab176647), tumor necrosis factor (TNF)- $\alpha$ (cat. no. ab100747), interleukin (IL)-6 (cat. no. ab100712), chemokine (C-X-C motif) ligand 1 (Cxcl-1) (cat. no. ab216951) (all from Abcam, Cambridge, MA, USA) and TLR-4 (cat. no. ABIN424269; antibodies-online Inc., Atlanta, GA, USA). Procedures were conducted following the manufacturer's protocol and the final results were measured at $450 \mathrm{~nm}$ using an ELISA plate reader.

Western blot analysis. Epithelial cells were isolated from berberine-treated mice, homogenized in a RIPA buffer (cat. no. R0278; Sigma-Aldrich; Merck KGaA, Darmstadt, Germany) containing protease-inhibitor and centrifuged at $3,000 \mathrm{x} \mathrm{g}$ at $4^{\circ} \mathrm{C}$ for $10 \mathrm{~min}$, and the protein concentration was measured by a BCA assay kit (cat. no. 23225; Thermo Fisher Scientific, Inc.). Proteins (20 $\mu \mathrm{g} / \mathrm{lane})$ were loaded for SDS-PAGE: The stacking gel concentration was $5 \%$ and separating gel was $12 \%$. The proteins were then transferred to a PVDF membrane (EMD Millipore, Billerica, MA, USA). All the primary antibodies except for anti-EGF were purchased from Cell Signaling Technology, Inc. (Danvers, MA, USA), including caspase3 (cat. no. 9662), caspase9 (cat. no. 9502), survivin (cat. no. 2808), Cyto $c$ (cat. no. 11940), caspase8 (cat. no. 4790), FADD (cat. no. 2782), c-Myc (cat. no. 13987), P53 (cat. no. 2524), IFN- $\gamma$ (cat. no. 8455), PI3K (cat. no. 4249), AKT (cat. no. 4691), pAKT (cat. no. 4060), pERK (cat. no. 3192), Bcl-2 (cat. no. 3498) (all 1:1,000) and GAPDH (cat. no. 5174; 1:2,000). Primary antibodies directed against EGF (cat. no. ab9695) was purchased from Abcam. Primary antibodies were incubated with the membranes at $4^{\circ} \mathrm{C}$ for overnight then blocked with $5 \%$ skimmed milk for $1 \mathrm{~h}$ at $37^{\circ} \mathrm{C}$. Subsequently, proteins were and incubated with corresponding horseradish peroxidase (HRP)-conjugated 
secondary anti-rabbit (cat. no. 7071) and anti-mouse (cat. no. 4410) antibodies (all 1:5,000; Cell Signaling Technology, Inc.) for $30 \mathrm{~min}$ at room temperature. Proteins were visualized using an enhanced chemiluminescence detection system (Pierce ECL Plus Western Blotting Substrate; Thermo Fisher Scientific, Inc.) and analyzed by ImageJ software (version 1.47; National Institutes of Health, Bethesda, MD, USA).

Flow cytometry. Apoptosis rates of the epithelial cells were evaluated using an Annexin V-fluorescein isothiocyanate (FITC) and propidium iodide (PI) apoptosis detection kit (BD Biosciences, San Jose, CA, USA). Epithelial cells were isolated from berberine- or PBS-treated mice and suspended with Annexin V-FITC and PI, following the manufacturer's instructions. Fluorescence was measured using a fluorescence activated cell sorting flow cytometer.

Cell viability assay. Epithelial cells $\left(2 \times 10^{3}\right.$ cells/well $)$ were isolated from berberine-treated mice and seeded in a 96 well microplate; the epithelial cells were cultured until they reached $90 \%$ confluence. The culture medium was then removed, epithelial cells were washed three times and subsequently incubated with $1 \%$ Triton $^{\mathrm{TM}}$ X-100 (cat. no. 9002-93-1; Sigma-Aldrich; Merck KGaA) for $30 \mathrm{~min}$ in room temperature. Lactate dehydrogenase activity in the lysates was measured using a CytoTox $96^{\circledR}$ assay kit (Promega Corporation) and a microplate reader at a wavelength of $490 \mathrm{~nm}$. All procedures were conducted following the manufacturer's instructions.

Analysis of physical activity by indirect calorimetry. Indirect calorimetry was performed to evaluate the physical activity of mice with neonatal NEC using the comprehensive laboratory animal monitoring system (Columbus Instruments International, Columbus, OH, USA). On day 10 after treatment with berberine, the physical activity of mice was monitored every $30 \mathrm{~min}$ for $24 \mathrm{~h}$.

Immunohistochemistry. Fluorescein conjugated staining was performed to analyze the apoptosis of small intestine cells and was conducted using the in situ Cell Death Detection kit (Roche Diagnostics, Basel, Switzerland), following the manufacturer's instruction. Paraffin-embedded epithelial tissue sections (4-5- $\mu$ m-thick) were fixed with $3.7 \%$ formalin at room temperature for $18-24 \mathrm{~h}$ and then the epitope retrieval was performed. The slides were incubated in 10X Antigen Retrieval Reagent-Basic (R\&D Systems, Inc., Minneapolis, $\mathrm{MN}, \mathrm{USA}$ ) at $92-95^{\circ} \mathrm{C}$ for $2-10 \mathrm{~min}$, cool in room temperature, rinsed with $\mathrm{ddH}_{2} \mathrm{O}$ and washed with PBS. Paraffin sections were exposed to hydrogen peroxide (3\%) for $10-15 \mathrm{~min}$ in room temperature and were then blocked using a regular blocking solution [StartingBlock (PBS) Blocking Buffer; Thermo Fisher Scientific, Inc.] for $10-15 \mathrm{~min}$ at $37^{\circ} \mathrm{C}$. In addition, sections were incubated with rabbit anti-mouse primary antibodies directed against EGF (cat. no. ab9695) or Bcl-2 (cat. no. ab59348) (both 1:200; Abcam) for $2 \mathrm{~h}$ at $37^{\circ} \mathrm{C}$ and washed three times with PBS. Subsequently, sections were incubated with HRP-conjugated goat anti-rabbit secondary antibodies (cat. no. ab6721, 1:1,000; Abcam) for $1 \mathrm{~h}$ at $37^{\circ} \mathrm{C}$. The TUNEL assay was performed using the TUNEL apoptosis detection kit (cat. no. L00297; GenScript, Piscataway Township, NJ,
USA) according to a manufacturer's instructions. Fluorescence Mounting medium was purchased from Trevigen, Inc. (Gaithersburg, MD, USA) and images were captured using nine fields of view. Finally, sections were stained with hematoxylin and eosin stain at $4^{\circ} \mathrm{C}$ for $12 \mathrm{~h}$. A Nikon Eclipse TE2000-U Inverted Microscope (Nikon Corporation, Tokyo, Japan) combined with Nikon optics was used to photograph all sections.

Statistical analysis. All data are presented as the mean \pm standard error of the mean. Unpaired data was analyzed using a Student's t test. Comparisons of data among multiple groups were analyzed by a two-way analysis of variance followed by Tukey's post hoc test. $\mathrm{P}<0.05$ was considered to indicate a statistically significant difference.

\section{Results}

Berberine decreases levels of inflammatory cytokines in the peripheral blood of mice with neonatal NEC. The anti-inflammatory effects of berberine in mice with neonatal NEC were investigated. Levels of TLR-4 (Fig. 1A), MD-2 (Fig. 1B), TNF- $\alpha$ (Fig. 1C), NF- $\kappa$ B (Fig. 1D), IL-6 (Fig. 1E) and Cxcl-1 (Fig. 1F) were all significantly decreased in the peripheral blood of mice treated with berberine compared with those treated with PBS. These results indicate that berberine significantly decreases inflammatory cytokine levels in the peripheral blood of mice with neonatal NEC.

Berberine inhibits the apoptosis of epithelial cells via the mitochondrial apoptosis pathway. The anti-apoptotic effects of berberine on epithelial cells in a mouse model of NEC were analyzed. Berberine significantly decreased the rate of apoptosis in epithelial cells compared with PBS-treated mice (Fig. 2A). Furthermore, expression of the pro-apoptosis genes caspase- 3 and -9 were significantly decreased in epithelial cells following the administration of berberine (Fig. 2B). It was also demonstrated that the expression of survivin and cytochrome $c$ were significantly upregulated in the epithelial cells of berberine-treated mice compared with PBS-treated mice; upregulated survivin and cytochrome $c$ may inhibit the apoptosis of epithelial cells (Fig. 2C). The expression of caspase- 8 and Fas-associated protein with death domain were not notably altered in the epithelial cells of berberine-treated mice compared with control mice (Fig. 2D). Furthermore, the expression of anti-apoptotic genes, including proto-oncogene c-Myc, tumor suppressor protein p53 and interferon (IFN)- $\gamma$ were significantly upregulated following treatment with berberine (Fig. 2E and F). These results indicate that berberine significantly inhibits the apoptosis of epithelial cells by downregulating the expression of proteins in the mitochondrial apoptosis pathway.

Berberine reduces the apoptosis of epithelial cells by influencing the PI3K/AKT signaling pathway. The molecular mechanism of berberine-mediated attenuation of epithelial cell apoptosis in a mouse model of NEC was investigated. Berberine treatment significantly inhibited the expression and phosphorylation of PI3K and AKT in epithelial cells compared with controls (Fig. 3A and B). Furthermore, EGF and Bcl-2 


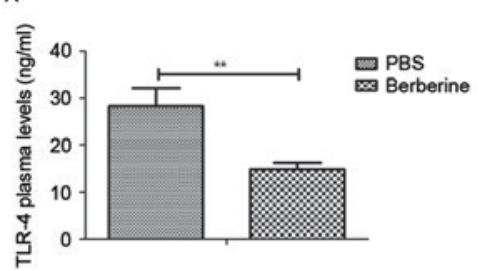

D

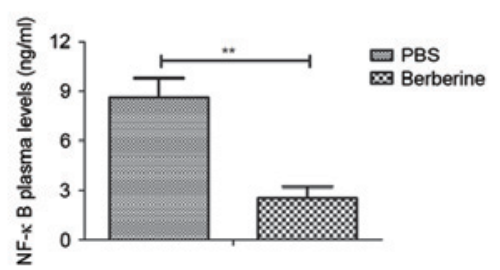

B

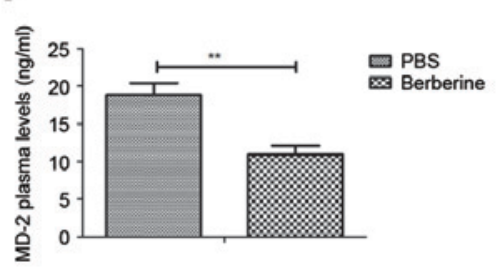

E

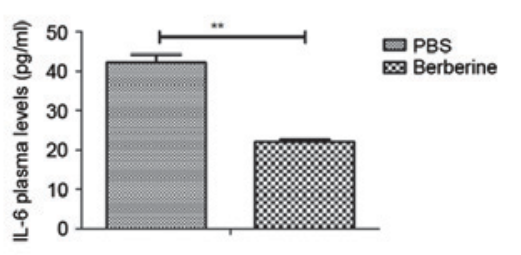

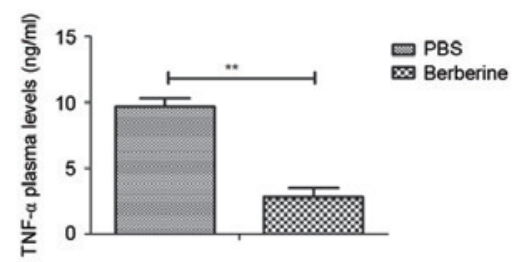

F

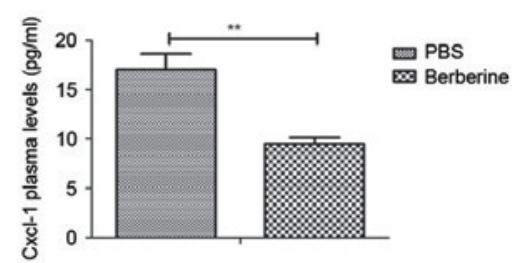

Figure 1. Berberine significantly reduces inflammatory cytokine levels in the peripheral blood of mice with neonatal NEC. Mice with neonatal NEC were treated with either berberine or PBS (as a control) and levels of (A) TLR-4, (B) MD-2, (C) TNF- $\alpha$, (D) NF- $\kappa$ B, (E) IL-6 and (F) Cxcl-1 in the peripheral blood was determined by ELISA. ${ }^{* *} \mathrm{P}<0.01$. NEC, necrotizing enterocolitis; TLR, toll like receptor; MD, myeloid differentiation protein; TNF, tumor necrosis factor; NF nuclear factor; IL, interleukin; Cxcl, (C-X-C motif) ligand.

A
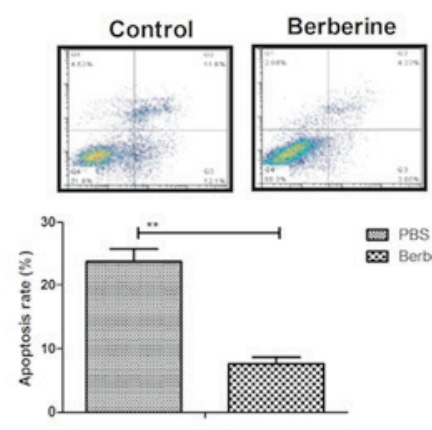

D

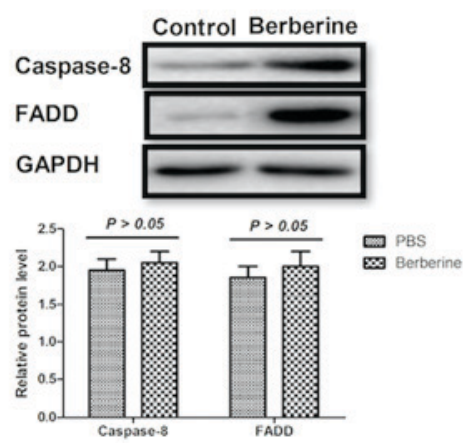

B
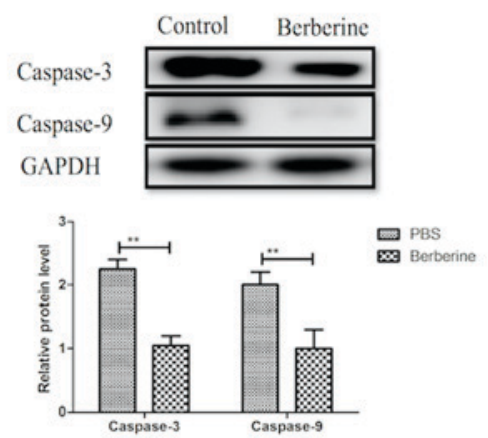

E
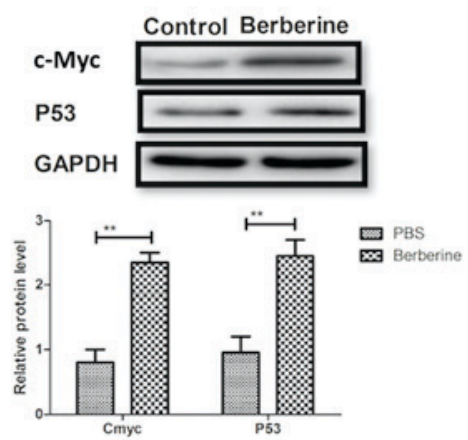

C
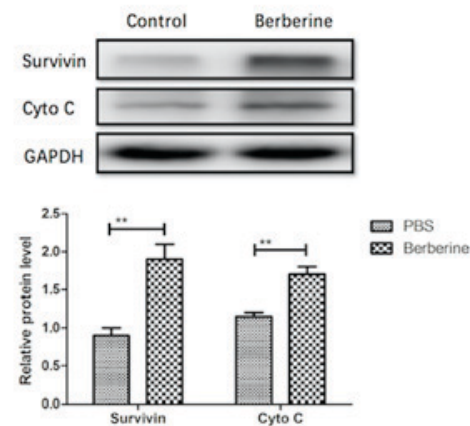

F
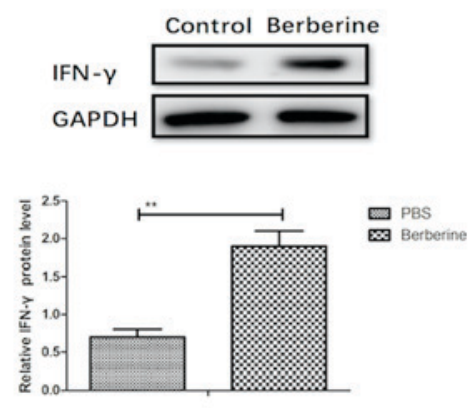

Figure 2. Berberine reduces the apoptosis of epithelial cells through the mitochondrial apoptosis pathway. (A) The apoptosis rate of epithelial cells in the small intestine was determined by flow cytometry. The protein expression levels of (B) caspase-3 and caspase-9, (C) survivin and Cyto $c$, (D) caspase- 8 and FADD, (E) c-Myc and p53 and (F) IFN- $\gamma$ in epithelial cells in the small intestine were determined by western blot analysis. ${ }^{* *} \mathrm{P}<0.01$. Cyto, cytochrome; FADD, Fas-associated protein with death domain; c-Myc, Myc proto-oncogene protein; p53, tumor suppressor protein p53; IFN, interferon.

expression was markedly upregulated in the epithelial cells of mice treated with berberine (Fig. 3C). The results of in vitro assays revealed that treatment with PI3K alone significantly promoted the apoptosis of epithelial cells isolated from mice with NEC compared with controls; however the apoptosis rate of cells treated with berberine did not differ significantly from that of the control (Fig. 3D). Furthermore, treatment with PI3K alone promoted the expression and phosphorylation of AKT; however, this effect was eliminated when PI3K and berberine were administered together (Fig. 3E). PI3K treatment alone significantly inhibited the expression of EGF and $\mathrm{Bcl}-2$, and berberine treatment significantly increased EGF and Bcl-2 expression in 
A
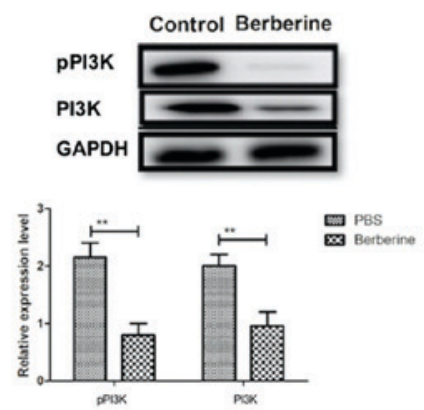

D
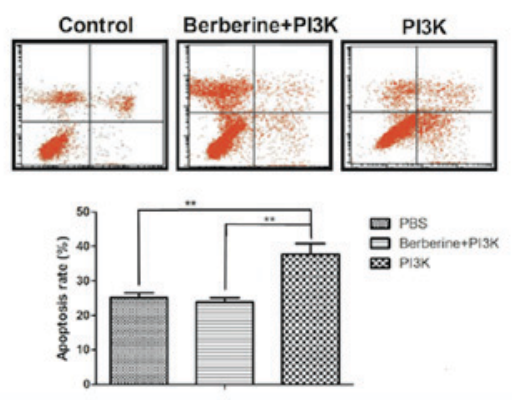

B
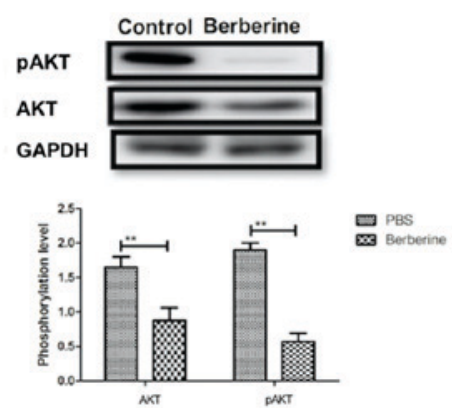

E
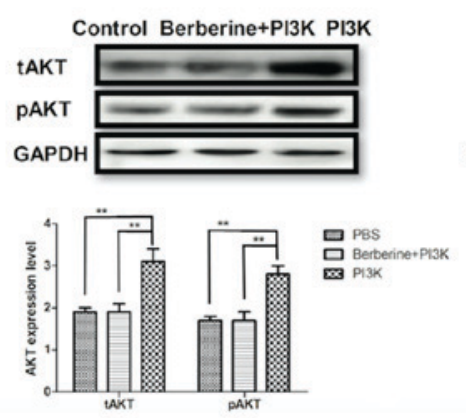

C
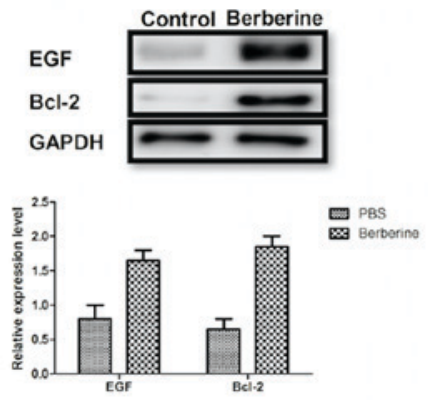

$\mathbf{F}$
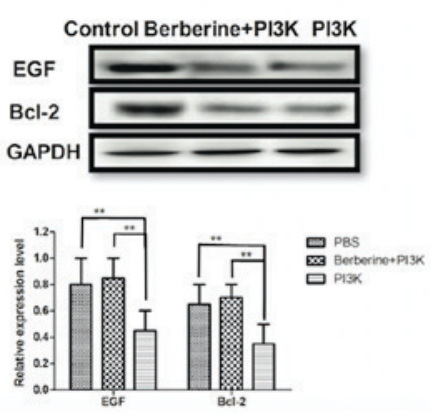

Figure 3. Berberine alleviates the apoptosis of epithelial cells by inhibiting the PI3K/AKT signaling pathway. Western blot analysis was used to determine (A) the expression of pPI3K and PI3K, (B) the phosphorylation levels of pAKT and AKT and (C) the expression of EGF and Bcl-2 in epithelial cells following treatment with either berberine or PBS. (D) The apoptosis rate of epithelial cells following treatment with either PI3K, PI3K+berberine or PBS alone was determined using flow cytometry. (E) The expression and phosphorylation levels of AKT and (F) the expression of EGF and Bcl-2 in epithelial cells following treatment with either PI3K, PI3K+berberine or PBS was determined by western blot analysis. tAKT served as the control. ${ }^{* *} \mathrm{P}<0.01$. PI3K, phosphoinositide 3-kinase; AKT, protein kinase B; EGF, epithelial growth factor; Bcl, B-cell lymphoma; p, phosphorylated; tAKT, total AKT.

A

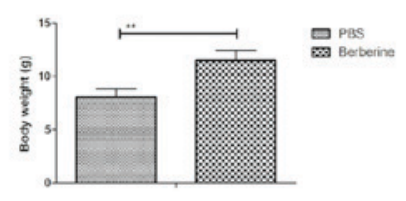

D

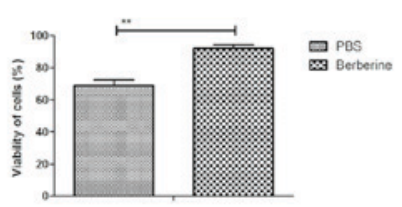

B

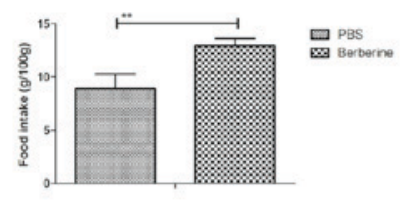

E

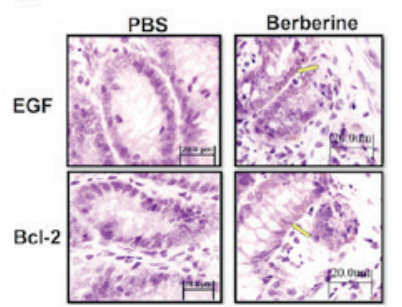

C

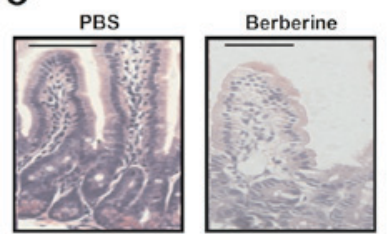

$\mathrm{F}$

PBS

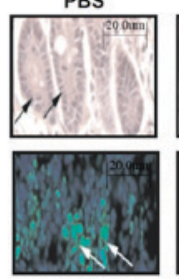

Berberine
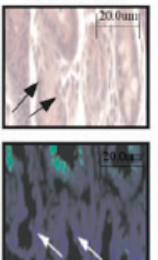

Figure 4. Berberine improves the physical activity and physiological functions of mice with neonatal NEC. The (A) body weight and (B) food intake of mice with NEC following treatment with berberine or PBS was determined by physical activity analysis. (C) The area of infarction in the small intestine of mice with neonatal NEC was determined by staining with hematoxylin and eosin. (D) The viability of epithelial cells following treatment with berberine treatment was determined by a viability assay. (E) EGF and Bcl-2 expression in the small intestine were determined by immunohistochemistry. (F) The apoptosis of epithelial cells in the small intestine was determined by a TUNEL assay. The arrows pointed to the positive sites. ${ }^{* *} \mathrm{P}<0.01$. NEC, necrotizing enterocolitis; EGF, epithelial growth factor; Bcl, B-cell lymphoma; TUNEL, terminal deoxynucleotidyl transferase dUTP nick-end labeling.

epithelial cells compared to PI3K treatment (Fig. 3F). These results suggest that berberine reduces the apoptosis of epithelial cells by mediating the PI3K/AKT signaling pathway.

Berberine improves the physical activity and physiological functions of mice with neonatal NEC. To analyze the efficacy of berberine in a mouse model of neonatal NEC, the effect of berberine on the physical activity and physiological functions of mice was investigated. The body weight (Fig. 4A) and food intake (Fig. 4B) of mice treated with berberine were significantly increased compared with untreated mice with neonatal NEC. Berberine treatment notably decreased the area of infarction 
in the small intestine compared with the PBS control group (Fig. 4C). The viability of epithelial cells was also significantly increased in mice treated with berberine compared with those treated with PBS (Fig. 4D). In addition, treatment with berberine markedly improved the expression of EGF and Bcl-2, as determined by histological analysis (Fig. 4E). Immunohistochemistry indicated that the apoptosis rate of epithelial cells was also notably decreased following treatment with berberine compared with the control (Fig. 4F). These results indicate that berberine improves the physical activity and physiological functions in a mouse model of neonatal NEC.

\section{Discussion}

NEC is an acquired disease that occurs in immature small intestinal tissues and is caused by an insufficient blood supply, dietary disorders and bacterial infection (20). The poor prognosis of individuals with neonatal NEC contributes to the high mortality rate of premature babies. At present, methods of treating NEC ameliorate necrosis in the small intestine and aim to attenuate its destructive progression (21). The pathogenesis of NEC is not yet comprehensively understood; therefore the treatment options available are not specifically targeted, meaning that they are not particularly effective and that there is potential for improvement. In the present study, the potential mechanisms by which treatment with berberine had a positive effect on NEC in a mouse model were explored. The anti-inflammatory and anti-apoptotic effects of berberine in a mouse model of neonatal NEC were determined. The results demonstrated that berberine treatment significantly downregulated inflammation and apoptosis while markedly improving the physical activity and physiological functions of mice. Notably, the results indicated that berberine treatment inhibits inflammation and apoptosis by inhibiting the PI3K/AKT signaling pathway.

Inflammation serves an essential role in the development of neonatal NEC (22). Previous studies have reported that preventing inflammation is an effective method of protecting against myocardial ischemia-reperfusion injury and have highlighted it as a novel technique of diagnosing and treating patients with cardiac disease $(23,24)$. Loubele et al (24) reported that the inhibition of apoptosis and inflammation contributes to the rehabilitation of myocardial ischemia/reperfusion injury. In addition, Wang et al (25) demonstrated that berberine acts as anti-arrhythmic drug in a rat type II diabetic myocardial infarction model by repressing the inward rectifier potassium channel 2. Berberine also exhibits anti-inflammatory effects on patients with acute coronary syndrome that have experienced percutaneous coronary intervention (26). Additionally, berberine prevents postsurgical intestinal adhesion and inhibits inflammation in a rat model by inhibiting the transforming growth factor $\beta$-activated kinase 1 (TAK1)/Jun $\mathrm{N}$-terminal kinase and TAK1/NF- $\mathrm{B}$ signaling pathways (22). Therefore, berberine may regulate inflammation in the progression of neonatal NEC via the PI3K/AKT signaling pathway. These results indicate that berberine significantly reduces levels of the inflammatory cytokines TLR4, MD-2, TNF- $\alpha, N F-\kappa B$, IL- 6 and Cxcl-1 in the epithelial cells of a mouse model of neonatal NEC. These cytokines contribute to the activity of epithelial cells and when they are decreased, the apoptosis rate of the cells also decreases. Berberine is able to reduce the apoptosis of epithelial cells in a mouse model of neonatal NEC via this mechanism.

Neonatal NEC is characterized as the extensive apoptosis and necrosis of the intestinal epithelium. It has been reported that inhibitors of apoptosis can regulate intestinal immunity and inflammation, and ameliorate bowel diseases (21). Additionally, inhibiting $\mathrm{NF}-\kappa \mathrm{B}$ expression improves resolution of mucosal inflammation in the intestinal epithelium (20). Furthermore, it has been demonstrated that berberine inhibits ischemia-induced apoptosis by activating the PI3K/PKB signaling pathway (22). Therefore, various strategies aiming at preventing or mitigating the extent of apoptosis have been performed to try to protect the small intestine against apoptosis or necrosis $(23,27,28)$. In the present study, it was hypothesized that berberine may regulate the apoptosis of epithelial cells in a mouse model of neonatal NEC by regulating the PI3K/AKT signaling pathway. The results confirm this hypothesis and reveal that berberine suppresses the mitochondrial apoptosis pathway in epithelial cells by inhibiting the PI3K/AKT signaling pathway.

In conclusion, the present study investigated the potential therapeutic effects of berberine and its ability to protect the small intestine against necrosis. It was demonstrated that berberine reduces epithelial cell apoptosis and tissue necrosis by inhibiting inflammation and downregulating the PI3K/AKT signaling pathway. The therapeutic effect of berberine during recovery may be attributed to its upregulation of EGF and Bcl-2 expression. The results also indicated that physical activity and physiological functions of mice with neonatal NEC were improved following treatment with berberine, suggesting that berberine may be a potential novel therapeutic agent for the treatment of neonatal NEC.

\section{References}

1. Barak S, Riskin A, Kugelman A, Abend-Weinger M, Chistyakov I and Bader D: Necrotizing enterocolitis in a premature infant as the presenting symptom of familial dysautonomia in the neonatal period: Case report and review of the literature. Am J Perinatol 22: 353-355, 2005.

2. Claud EC: Neonatal Necrotizing enterocolitis-inflammation and intestinal immaturity. Antiinflamm Antiallergy Agents Med Chem 8: 248-259, 2009.

3. Demirbag S: Peer review report 1 on 'Gene expression profile of necrotizing enterocolitis model in neonatal mice'. Int J Surg 13 (Suppl 1): S183, 2015.

4. Sharma D and Shastri S: Lactoferrin and neonatology-role in neonatal sepsis and necrotizing enterocolitis: Present, past and future. J Maternal Fetal Neonatal Med 29: 763-770, 2016.

5. Egan CE, Sodhi CP, Good M, Lin J, Jia H, Yamaguchi Y, Lu P, Ma C, Branca MF, Weyandt S, et al: Toll-like receptor 4-mediated lymphocyte influx induces neonatal necrotizing enterocolitis. J Clin Invest 126: 495-508, 2016.

6. Ginzel M, Yu Y, Klemann C, Feng X, von Wasielewski R, Park JK, Hornef MW, Torow N, Vieten G, Ure BM, et al: The viral dsRNA analogue poly (I:C) induces necrotizing enterocolitis in neonatal mice. Pediatr Res 79: 596-602, 2016.

7. Caplan MS, Russell T, Xiao Y, Amer M, Kaup S and Jilling T: Effect of polyunsaturated fatty acid (PUFA) supplementation on intestinal inflammation and necrotizing enterocolitis (NEC) in a neonatal rat model. Pediatr Res 49: 647-652, 2001.

8. Nanthakumar NN, Fusunyan RD, Sanderson I and Walker WA: Inflammation in the developing human intestine: A possible pathophysiologic contribution to necrotizing enterocolitis. Proc Natl Acad Sci USA 97: 6043-6048, 2000.

9. Chan KL, Wong KF and Luk JM: Role of LPS/CD14/ TLR4-mediated inflammation in necrotizing enterocolitis: Pathogenesis and therapeutic implications. World J Gastroenterol 15: 4745-4752, 2009. 
10. Guner YS, Franklin AL, Chokshi NK, Castle SL, Pontarelli E, Wang J, Wang L, Prasadarao NV, Upperman JS, Grishin AV and Ford HR: P-glycoprotein induction by breast milk attenuates intestinal inflammation in experimental necrotizing enterocolitis. Lab Invest 91: 1668-1679, 2011.

11. Arciero JC, Ermentrout GB, Upperman JS, Vodovotz Y and Rubin JE: Using a mathematical model to analyze the role of probiotics and inflammation in necrotizing enterocolitis. PloS One 5: e10066, 2010.

12. Jiang Q, Liu P, Wu X, Liu W, Shen X, Lan T, Xu S, Peng J, Xie X and Huang H: Berberine attenuates lipopolysaccharide-induced extracelluar matrix accumulation and inflammation in rat mesangial cells: Involvement of NF- $\kappa \mathrm{B}$ signaling pathway. Mol Cell Endocrinol 331: 34-40, 2011.

13. Huang LG, Zhou W, Rong X, Tao L and Lu WN: Effects of glycomacropeptide on damage to intestinal tissue and apoptosis of intestinal epithelial cells in neonatal rats with necrotizing enterocolitis. Zhonghua Er Ke Za Zhi 50: 536-542, 2012 (In Chinese).

14. Jilling T, Lu J, Jackson M and Caplan MS: Intestinal epithelial apoptosis initiates gross bowel necrosis in an experimental rat model of neonatal necrotizing enterocolitis. Pediatr Res 55: 622-629, 2004

15. Clark JA, Lane RH, Maclennan NK, Holubec H, Dvorakova K, Halpern MD, Williams CS, Payne CM and Dvorak B: Epidermal growth factor reduces intestinal apoptosis in an experimental model of necrotizing enterocolitis. Am J Physiol Gastrointest Liver Physiol 288: G755-G762, 2005.

16. Feng J, El-Assal ON and Besner GE: Heparin-binding epidermal growth factor-like growth factor reduces intestinal apoptosis in neonatal rats with necrotizing enterocolitis. J Pediatr Surg 41: 742-747, 2006.

17. Chen K, Li G, Geng F, Zhang Z, Li J, Yang M, Dong L and Gao F: Berberine reduces ischemia/reperfusion-induced myocardial apoptosis via activating AMPK and PI3K-Akt signaling in diabetic rats. Apoptosis 19: 946-957, 2014

18. Medical Ethics Committee of Zhongnan Hospital of Wuhan University. Medical ethics committee (Internet). http://www. znhospital.cn/llwyh/4882.jhtml. Accessed October 28, 2017.

19. Jung K, Kim JH, Cheong HS, Shin E, Kim SH, Hwang JY, Lee E, Yoon MO, Kim SH, Sio CA, et al: Gene expression profile of necrotizing enterocolitis model in neonatal mice. Int J Surg 23: $28-34,2015$.
20. Hammers AL, Sanchez-Ramos L and Kaunitz AM: Antenatal exposure to indomethacin increases the risk of severe intraventricular hemorrhage, necrotizing enterocolitis, and periventricular leukomalacia: A systematic review with metaanalysis. Am J Obste Gynecol 212: 505.e1-13, 2015.

21. Pedersen J, LaCasse EC, Seidelin JB, Coskun M and Nielsen OH: Inhibitors of apoptosis (IAPs) regulate intestinal immunity and inflammatory bowel disease (IBD) inflammation. Trends Mol Med 20: 652-665, 2014.

22. Kim M, Shin MS, Lee JM, Cho HS, Kim CJ, Kim YJ, Choi HR and Jeon JW: Inhibitory effects of Isoquinoline Alkaloid Berberine on ischemia-induced apoptosis via activation of phosphoinositide 3-kinase/protein kinase B signaling pathway. Int Neurourol J 18: 115-125, 2014.

23. Liu LL, Lin LR, Lu CX, Fu JG, Chao PL, Jin HW, Zhang ZY and Yang TC: Expression of inflammatory and apoptosis factors following coronary stent implantation in coronary heart disease patients. Int Immunopharmacol 11: 1850-1854, 2011.

24. Loubele ST, Spek CA, Leenders P, van Oerle R, Aberson HL, Hamulyák K, Ferrell G, Esmon CT, Spronk HM and ten Cate $\mathrm{H}$ : Activated protein $\mathrm{C}$ protects against myocardial ischemia/reperfusion injury via inhibition of apoptosis and inflammation. Arterioscler Thromb Vasc Biol 29: 1087-1092, 2009.

25. Wang $\mathrm{LH}, \mathrm{Yu} \mathrm{CH}, \mathrm{Fu}$ Y, Li Q and Sun YQ: Berberine elicits anti-arrhythmic effects via IK1/Kir2.1 in the rat type 2 diabetic myocardial infarction model. Phytother Res 25: 33-37, 2011.

26. Zhang Y, Li X, Zhang Q, Li J, Ju J, Du N, Liu X, Chen X, Cheng F, Yang L, et al: Berberine hydrochloride prevents postsurgery intestinal adhesion and inflammation in rats. J Pharmacol Exp Ther 349: 417-426, 2014.

27. Salmina AB, Shul'man VA, Nikulina SY, Trufanova LV, Fursov AA, But'yanov PA, Kuskaev AP, Bol'shakova EV and Kotlovskii MY: Apoptosis of leukocytes as a marker of neutrophil-endotheliocyte interaction in coronary heart disease. Bull Exp Biol Med 144: 39-41, 2007 (In English, Russian).

28. Geng YJ: Molecular mechanisms for cardiovascular stem cell apoptosis and growth in the hearts with atherosclerotic coronary disease and ischemic heart failure. Ann N Y Acad Sci 1010: 687-697, 2003. 